\title{
Attack on the Cryptographic Scheme NIKS-TAS
}

\author{
Don Coppersmith
}

IBM Research, T.J. Watson Research Center
Yorktown Heights, NY 10598, USA

\begin{abstract}
The NIKS-TAS scheme, proposed by Tsujii, Araki, and Sekine in 1993, is an ID-based cryptographic key sharing scheme. We present an algebraic method for attacking this scheme, requiring the cooperation of a small number of collaborators to discover the key shared by any two parties.
\end{abstract}

\section{Introduction}

The NIKS-TAS scheme, proposed by Tsujii, Araki, and Sekine [2] in 1993, is an ID-based cryptographic key sharing scheme. That is, to each user (User $A$ ) there corresponds a publicly known quantity $I D_{A}$, closely related to his personal identification; for example, this could consist of name, address, and telephone number. User $A$ also owns some secret information, which we temporarily abbreviate $\mathrm{X}_{A}$. Suppose Users $A$ and $B$ wish to communicate secretly. User $A$ executes the NIKS-TAS protocol, using as inputs his own secret information $\mathrm{X}_{A}$ and User $B^{\prime}$ s public information $I D_{B}$, to prepare a cryptographic key $K_{A B}=f\left(\mathrm{X}_{A}, I D_{B}\right)$. Simultaneously, User $B$ uses $\mathrm{X}_{B}$ and $I D_{A}$ to compute the key $K_{B A}=f\left(\mathrm{X}_{B}, I D_{A}\right)$. The NIKS-TAS scheme is designed such that $K_{A B}=K_{B A}$. Thus both $A$ and $B$ can compute the same cryptographic key, and use it in private communication with each other.

The scheme shares some aspects with the Diffie and Hellman key exchange scheme [1], but differs in one crucial place. In the Diffie and Hellman scheme, public information includes a large prime $p$ and a generator $g$ of the multiplicative group of integers modulo $p$, called $Z_{p}{ }^{*}$. The user's public information $y_{A}$ is a member of the multiplicative group $Z_{p}{ }^{*}$, while the secret information $x_{A}$ lies in the additive group of exponents or logarithms. The security lies in the fact that computing a group element from its logarithm is easy, but computing the logarithm from the group element seems to be difficult. 


$$
\begin{aligned}
\text { public: } & y_{A} \in \mathbf{Z}_{p}{ }^{*} \\
\text { secret: } & x_{A} \in Z_{p-1} \\
& y_{A}=g^{\left(x_{A}\right)} \in \mathbf{Z}_{p}{ }^{*} \\
\text { easy: } & x_{A} \rightarrow y_{A} \\
\text { hard: } & y_{A} \rightarrow x_{A}
\end{aligned}
$$

By contrast, in the NIKS-TAS scheme the public information is used to operate in the additive group of exponents, and some of the secret information lies in the multiplicative group $Z_{p}{ }^{*}$.

$$
\begin{aligned}
\text { public: } & I D_{A} \rightarrow i_{A, j, 1} \in\{0,1\} \text { operating on } Z_{p-1} \\
\text { secret: } & \mathrm{X}_{A} \rightarrow G_{A k} \in \mathbf{Z}_{p}{ }^{*}
\end{aligned}
$$

This reversal comes about because of the requirements of ID-based cryptography. For example, if one tried to make the Diffie-Hellman scheme ID-based, the public information $y_{A}$ would be predetermined (by the user's name, etc.) and someone (a trusted key distribution center, perhaps) would have to derive the secret information $x_{A}$ from this public information $y_{A}$. But the premise of the Diffie-Hellman scheme is that this task is computationally infeasible, even for the key distribution center. So, in the NIKS-TAS, in order to accommodate the ID-based requirements, the roles are reversed.

When the NIKS-TAS scheme was announced, its inventors offered a reward of $\$ 3,000$ (US) for showing "theoretically substantial defects proving that our scheme cannot overcome the collusion problem." The present paper represents a cryptanalysis of this scheme and an attempt to claim the reward.

We show here that a small number of colluding users can pool their secret information and obtain enough knowledge that they can easily find the key shared by any two other users. For a typical value of the parameters of the system, $N=20,43$ users can usually conspire and break the system.

The attack on the system is made possible by the reversal of roles from the Diffie-Hellman system: the fact that the public information is involved in the ring $Z_{p-1}$ instead of the multiplicative group $Z_{p}{ }^{*}$. Where the legitimate user computes a key in $\mathrm{Z}_{p}{ }^{*}$, the attacker mirrors this computation with computations in the group of exponents $Z_{p-1}$, where he has greater flexibility in the computations available to him, and thus is able to carry out an attack not en- 
visioned by the system's designers. The attack uses differential techniques: User $A$ considers keys $K_{A B}$ and $K_{A B}$, which he would share with two users $B$ and $B^{\prime}$ whose public information $I D_{B}$ and $I D_{B^{\prime}}$ differ in only one bit, and uses the difference between $K_{A B}$ and $K_{A B^{\prime}}$ to probe the secret information that should be inaccessible to him.

In the balance of the paper, we present the NIKS-TAS scheme, condensed from [2], followed by our attack, and a worked example.

\section{The NIKS-TAS Scheme}

This description is copied from [2], except that indices have been reordered for ease of understanding (particularly on the quantities $i_{A, j, m}$ ), and background material has been omitted.

\section{Public information common for all entities}

The trusted key distribution center publishes a large prime number $p$ such that the discrete logarithm problem $(\bmod p)$ is inaccessible. It determines the parameters of the system, $£, N$, and $L$; although not spelled out in the proposal, we can imagine $N=20, L=50$ and $f=200$. The center chooses and publishes a one-way hash function which sends $£$ bits to $K=N L$ bits.

\section{Public information for a single entity}

Each entity, User $A$, publicizes his $f$-bit IDCODE (which may be name and address) to all other entities, and registers the IDCODE at a trusted key distribution center. This code is processed by the publicly known hash function to obtain the $K$-bit ID vector of a typical entity $A$, called $I D_{A}$ :

$$
\begin{aligned}
& I D_{A}=\operatorname{Hash}\left(I D C O D E_{A}\right) \\
& I D_{A}=\left[i_{A, 1,1}, i_{A, 1,2}, \ldots, i_{A, N, L}\right]=\left[\mathbf{i}_{A 1}, \mathbf{i}_{A 2}, \ldots, \mathbf{i}_{A N}\right] \\
& i_{A, j, m} \in\{0,1\}, \quad j=1, \ldots, N, m=1, \ldots, L \\
& \mathbf{i}_{A j} \in\{0,1\}^{L}, \quad j=1, \ldots, N \\
& \mathbf{i}_{A j}=\left[i_{A, j, 1}, i_{A, j, 2}, \ldots, i_{A, j, L}\right]
\end{aligned}
$$

This data is publicly known, derived from the $f$-bit IDCODE by the public hash function. 


\section{Center Secrets common for all entities}

Meanwhile, the key distribution center chooses the following integers as secret parameters of its own:

$$
\begin{gathered}
g: \text { a generator of } \mathbf{Z}_{p}{ }^{*} \\
y \in \mathbf{Z}_{p-1} \\
\mathbf{x}_{j}, \mathbf{y}_{j}, \mathbf{u}_{j}, \mathbf{v}_{j} \in \mathbf{Z}_{p-1}^{L}, \quad j=1,2, \ldots, N \\
X=\left[x_{1,1}, x_{1,2}, \ldots, x_{N, L}\right]=\left[\mathbf{x}_{1}, \mathbf{x}_{2}, \ldots, \mathbf{x}_{N}\right] \\
Y=\left[y_{1,1}, y_{1,2}, \ldots, y_{N, L}\right]=\left[\mathbf{y}_{1}, \mathbf{y}_{2}, \ldots, \mathbf{y}_{N}\right] \\
U=\left[u_{1,1}, u_{1,2}, \ldots, u_{N, L}\right]=\left[\mathbf{u}_{1}, \mathbf{u}_{2}, \ldots, \mathbf{u}_{N}\right] \\
V=\left[v_{1,1}, v_{1,2}, \ldots, v_{N, L}\right]=\left[\mathbf{v}_{1}, \mathbf{v}_{2}, \ldots, \mathbf{v}_{N}\right]
\end{gathered}
$$

where $Z_{p-1}^{L}$ designates the set of $L$-dimensional vectors whose components belong to $\mathbf{Z}_{p-1} \cdot \mathbf{x}_{j}, \mathbf{y}_{j}, \mathbf{u}_{j}$, and $\mathbf{v}_{j}$ are global parameters with which the individual secret keys are generated.

\section{Individual secrets kept in center}

The center produces random numbers for each entity; namely the center produces $\alpha_{A j \ell}, \beta_{A j \ell}, c_{A k}$, and $r_{A j \ell}$ for entity $A$.

$$
\begin{aligned}
\alpha_{A j \ell}, \beta_{A j \ell} & \in \mathbf{Z}_{p-1}^{L}, \quad j=1,2, \ldots, N, \quad \ell=1,2, \ldots, 2(N+1-j) \\
{ }^{C} A k & \in Z_{p-1}, \quad k=1,2, \ldots, N+1 \\
r_{A j \ell} \in Z_{p-1}, & j=1,2, \ldots, N, \quad \ell=1,2, \ldots, 2(N+1-j)
\end{aligned}
$$

subject to the following relations:

$$
\begin{aligned}
& c_{A, i}^{(0)}=c_{A, i}, \quad i=1,2, \ldots, N+1 \\
& c_{A, i}^{(k)}=c_{A, i}^{(k-1)} r_{A, k, 2 i-1}+c_{A, i+1}^{(k-1)} r_{A, k, 2 i}, \quad k=1,2, \ldots, N, \quad i=1,2, \ldots, N-k+1 \\
& c_{A, i}^{(k-1)} \alpha_{A, k, 2 i-1}+c_{A, i+1}^{(k-1)}{ }_{A, k, 2 i}=0, \quad k=1,2, \ldots, N, \quad i=1,2, \ldots, N-k+1 \\
& c_{A, i}^{(k-1)} \beta_{A, k, 2 i-1}+c_{A, i+1}^{(k-1)} \beta_{A, k, 2 i}=0, \quad k=1,2, \ldots, N, \quad i=1,2, \ldots, N-k+1 \\
& \gamma=c_{A 1}^{(N)}
\end{aligned}
$$


The center calculates the following parameters for each entity in secret and keeps these parameters out of reach of any entity:

$$
\begin{aligned}
& S_{A j}=\mathbf{x}_{j} \cdot \mathbf{i}_{A j}+\mathbf{y}_{j} \cdot \mathbf{i}_{A j}^{c} \\
& T_{A j}=\mathbf{u}_{j} \cdot \mathbf{i}_{A j}+\mathbf{v}_{j} \cdot \mathbf{i}_{A j}^{c}
\end{aligned}
$$

where $i_{A j}^{c}$ is the complement of $i_{A j}$, and $\mathbf{x}_{j} \cdot \mathbf{i}_{A j}$ is the inner product.

\section{Individual secrets distributed to each entity}

The center calculates the following parameters for each entity, then distributes to each entity his own secret parameters through a secure channel:

$$
\begin{aligned}
\pi_{A} & \in S_{N} \quad \text { (a permutation) } \\
G_{A k} & =g^{c}{ }^{A k}, \quad k=1, \ldots, N+1 \\
\mathbf{X}_{A j \ell} & =r_{A j \ell}\left(S_{A j^{\prime}} \mathbf{x}_{j^{\prime}}+T_{\left.A j^{\prime} \mathbf{u}_{j^{\prime}}\right)+\alpha_{A j \ell}}\right. \\
\mathbf{Y}_{A j \ell} & =r_{A j \ell}\left(S_{A j^{\prime}} \mathbf{y}_{j^{\prime}}+T_{A j^{\prime} \mathbf{j}^{\prime}}\right)+\beta_{A j \ell} \\
& j=1,2, \ldots, N, \quad \ell=1,2, \ldots, 2(N+1-j)
\end{aligned}
$$

Here $\pi_{A}$ is a permutation on $N$ elements, and we define $j^{\prime}=\pi_{A}(j)$.

\section{Key Sharing Algorithm}

Two users $A$ and $B$ each compute a common key. User $A$ sets

$$
G_{A, B, i}^{(0)}=G_{A, i}, i=1, \ldots, N+1 .
$$

For each $k=1,2, \ldots, N$ in turn, User $A$ calculates:

$$
\begin{aligned}
& Z_{A, k, j}^{(B)}=\mathbf{X}_{A, k, j} \cdot \mathbf{i}_{B k^{\prime}}+\mathbf{Y}_{A, k, j} \cdot \mathbf{i}_{B k^{\prime}}^{c}, \quad j=1,2, \ldots, 2(N+1-k) \\
& =r_{A, k, j}\left(S_{A k^{\prime}} S_{B k^{\prime}}+T_{A k^{\prime}} T_{B k^{\prime}}\right)+\alpha_{A k j} \cdot \mathbf{i}_{B k^{\prime}}+\beta_{A k j} \cdot \mathbf{i}_{B k^{\prime}}^{C} \\
& G_{A, B, i}^{(k)}=\left(G_{A, B, i}^{(k-1)}\right)^{\left(Z_{A, k, 2 i-1}^{(B)}\right)} \times\left(G_{A, B, i+1}^{(k-1)}\right)^{\left(Z_{A, k, 2 l}^{(B)}\right)} \\
& =g^{\left\{c_{A, i}^{(k)} \Pi_{j=1}^{k}\left(S_{A j^{\prime}} S_{B j^{\prime}}+T_{A j^{\prime}} T_{B j^{\prime}}\right)\right\}}, \quad i=1,2, \ldots,(N-k+1) .
\end{aligned}
$$

Verifying these equalities is an exercise in algebra, and depends on the relations among the quantities $\alpha$, etc. 
After the Nth step we have

$$
K_{A B}=G_{A, B, 1}^{(N)}=g^{\left\{y \Pi_{j=1}^{N}\left(S_{A j} S_{B j}+T_{A j} T_{B j}\right)\right\}},
$$

recalling $\gamma=c_{A, 1}^{(N)}=c_{B, 1}^{(N)}$.

Meanwhile User $B$ performs analogous calculations using his secret quantities and $A^{\prime}$ s public quantities, to produce $K_{B A}$. One can check [2] that the two keys computed by these two users are the same:

$$
K_{A B}=K_{B A}
$$

User $A$ and User $B$ can use this common quantity $K_{A B}=K_{B A}$ as a cryptographic key for secret communication with each other.

\section{The Attack}

We show how $2 N+3$ collaborators can usually break the scheme. If $N=20$, then $M=43$ collaborators should suffice.

Notation: Users $A, B$ will be collaborators, and users $D, E, F$ will be victims. We will use public and secret information from the collaborators $A, B$, and we will use only public information from the victims $D, E, F$; in fact, we will fabricate public information for $D, E, F$ (invent new users with new public information, but no secret information) when it suits our purpose.

For any collaborator $A$ and user $E$ (who may be victim or collaborator), let $A$ follow the computation of key $K_{A E}$ from $G_{A k}=g^{c} A k, 1 \leq k \leq N+1$. Instead of computing $K_{A E^{\prime}} \quad A$ will compute exponents $e_{A E}^{(k)} \in \mathrm{Z}_{p-1}, \quad 1 \leq k \leq N+1$, such that

$$
K_{A E}=\prod_{k=1}^{N+1}\left(G_{A k}\right)^{e_{A E}^{(k)}}=g^{\left\{\Sigma_{k} c_{A k} e_{A E}^{(k)}\right\}}
$$

He does this by keeping track of powers of $G_{A k}$ in the computation of $K_{A E}$. Each $e_{A E}^{(k)}$ is a sum of products of the quantities $Z_{A, j, \ell}^{(E)}$.

Of special interest is the case where both $A$ and $B=E$ are collaborators. In this case, from the requirement $K_{A B}=K_{B A}$ we get: 


$$
\sum_{k=1}^{N+1} c_{A k} e_{A B}^{(k)}=\sum_{k=1}^{N+1} c_{B k} e_{B A}^{(k)}
$$

in the ring $Z_{p-1}$. The $e_{A B}^{(k)}$ are known, while $c_{A k}$ are unknown.

$\Lambda$ s we let $\{A, B\}$ range over all unordered pairs of collaborators, we obtain $M(M-1) / 2$ such linear equations $\left({ }^{*}\right)$, one for each unordered pair $\{A, B\}$. There are $M(N+1)$ unknowns $c_{A k}, 1 \leq k \leq N+1, A \in$ collaborators\}. Because we chose $M \geq 2 N+3$, we get $M(M-1) / 2 \geq M(N+1)$, so that there are at least as many equations as unknowns. These are homogeneous linear equations in $c_{A k}$. We solve them to get the ratios $c_{A k}: c_{B j}$. The example in the next section gives reason to believe that the rank of this system of homogeneous linear equations is $M(N+1)-1$, allowing for unique solution; and possible remedies for the opposite case.

Fix one collaborator $\underline{B}$ as a reference point, and set

$$
\hat{g}=G_{\underline{B} 1}=g^{c} \underline{B} 1 .
$$

We can calculate $\hat{g}$, and from now on we use it instead of the center's secret generator $g$, which we cannot calculate. We have gotten exponents $\hat{c}_{A k}$ such that

$$
G_{A k}=\hat{g}^{\hat{C}_{A k}}, \quad \forall A \in \text { collaborators }, \quad 1 \leq k \leq N+1 .
$$

Now we can work with these exponents $\hat{c}_{A k}$ and solve algebraic equations in the exponents.

Set $f_{A B}=\Sigma_{k} \hat{c}_{A k} e_{A B}^{(k)}$ so that $K_{A B}=\hat{g}^{f_{A B}}$, and $f_{A B}=f_{B A}$. We can calculate $f_{A B}$ if we know $A^{\prime}$ 's private information and $B^{\prime}$ s public information. ( $B$ need not be a collaborator; in fact we can fabricate $B^{\prime}$ s public information.)

Explanation. The data $\hat{c}_{A k}$ is gotten from the collaboration and from $A$ 's private information. The data $e_{A B}^{(k)}$ is gotten from $A$ 's private information and $B$ 's public information. So if $A$ is a collaborator and $E$ is a victim, $A$ can compute $f_{A E}$ such that $f_{A E}=\sum_{k} \hat{c}_{A k} e_{A E}^{(k)}$ and $K_{A E}=\hat{g}^{f} f_{A E}$.

We have 


$$
f_{A E}=\hat{\gamma} \prod_{j=1}^{N}\left(S_{A j} S_{E j}+T_{A j} T_{E j}\right)
$$

for $\hat{y}$ unknown, fixed, and independent of $A, E$. In fact, $\hat{y}=\gamma / c_{\underline{B} 1}$.

Now fix two collaborators, $A_{1}$ and $A_{2}$, and a victim, $D$. I et $A_{1}$ compute

$$
f_{A_{1} D}, f_{A_{1} D^{\prime}{ }_{1}}, f_{A_{1} D_{2}^{\prime}}, \ldots, f_{A_{1} D^{\prime}}{ }_{L}
$$

where $D_{j}^{\prime}$ is a fabricated user with the same public information as $D$ except for bit $i_{D 1 j}$. That is,

$$
\begin{aligned}
& i_{D_{j}^{\prime}, a, b}=i_{D, a, b} \text { if }(a, b) \neq(1, j) \\
& i_{D_{j}^{\prime}, 1, j}=1-i_{D, 1, j}
\end{aligned}
$$

Set $d_{A_{1} D 1}=\hat{\gamma} \pi_{i \neq 1}\left(S_{A_{1} i} S_{D i}+T_{A_{1} i} T_{D i}\right)$. As it happens, we do not know $d_{A_{1} D 1}$, but it is the same as $d_{A_{1} D_{j}^{\prime} 1}$. We can compute

$$
\pm\left(f_{A_{1} b_{j}^{\prime}}-f_{A_{1} D}\right)=d_{A_{1} D \mathrm{I}}\left\{S_{A_{1} 1}\left(x_{1 j}-y_{1 j}\right)+T_{A_{1} 1}\left(u_{1 j}-v_{1 j}\right)\right\}
$$

where $x_{1 j} \in \mathrm{Z}_{p-1}$ is the $j$ th entry of $\mathbf{x}_{1}$. The sign \pm is determined by the bit $i_{D, 1, j}$. By combining these values for the different $j$, we get some (unknown) linear combination of the two vectors $\left(x_{1}-y_{1}\right)$ and $\left(u_{1}-v_{1}\right)$; call it $q_{1}$ :

$$
\mathbf{q}_{1}=\left(d_{A_{1} D 1} S_{A_{1} 1}\right)\left(\mathbf{x}_{1}-\mathbf{y}_{1}\right)+\left(d_{A_{1} D 1} T_{A_{1} 1}\right)\left(\mathbf{u}_{1}-\mathbf{v}_{1}\right) \text {. }
$$

Repeat for another collaborator $A_{2}$ instead of $A_{1}$ to get a second (unknown) linear combination of $\left(x_{1}-y_{1}\right)$ and $\left(u_{1}-v_{1}\right)$; call this one $r_{1}$ :

$$
\mathbf{r}_{1}=\left(d_{A_{2} D 1} S_{A_{2} 1}\right)\left(\mathbf{x}_{1}-\mathbf{y}_{1}\right)+\left(d_{A_{2} D 1} T_{A_{2} 1}\right)\left(\mathbf{u}_{1}-\mathbf{v}_{1}\right) \text {. }
$$

Note: We can compute the vectors $\mathbf{q}_{1}$ and $r_{1}$, but not the quantities $d_{A_{1} E 1}, S_{A_{1} 1}, \mathbf{x}_{1}, \mathbf{y}_{1}, T_{A_{1} 1}, \mathbf{u}_{1}$, or $\mathbf{v}_{1}$.

The vectors $q_{1}, r_{1}$ span the same space as $\left(x_{1}-y_{1}\right)$ and $\left(u_{1}-v_{1}\right)$, a 2-dimensional subspace of $z_{p-1}^{L}$. We have computed $q_{1}$ and $r_{1}$. Put another way, we have computed the subspace spanned by $\left(x_{1}-y_{1}\right)$ and $\left(u_{1}-v_{1}\right)$.

Letting $A$ be an arbitrary collaborator and $E$ an arbitrary victim, we have that $\left(S_{A 1} S_{E 1}+T_{A 1} T_{E 1}\right)$ is an inhomogeneous bilinear form in 
$\left\{\left(\mathbf{x}_{1}-\mathbf{y}_{1}\right) \cdot \mathbf{i}_{A 1}, \quad\left(\mathbf{u}_{1}-\mathbf{v}_{1}\right) \cdot \mathbf{i}_{A 1}\right\} \quad$ (on the one hand) and $\left\{\left(\mathrm{x}_{1}-\mathrm{y}_{1}\right) \cdot \mathrm{i}_{E 1}, \quad\left(\mathrm{u}_{1}-\mathrm{v}_{1}\right) \cdot \mathrm{i}_{E 1}\right\}$ (on the other hand), namely

$$
\begin{aligned}
& \left(S_{A 1} S_{E 1}+T_{A 1} T_{E 1}\right)= \\
& \quad=\left[\left(\Sigma_{j} y_{1 j}\right)+\left(\mathbf{x}_{1}-\mathbf{y}_{1}\right) \cdot \mathbf{i}_{A 1}\right] \times\left[\left(\Sigma_{j} y_{1 j}\right)+\left(\mathbf{x}_{1}-\mathbf{y}_{1}\right) \cdot \mathbf{i}_{E 1}\right]+ \\
& \quad+\left[\left(\Sigma_{j} v_{1 j}\right)+\left(\mathbf{u}_{1}-\mathbf{v}_{1}\right) \cdot \mathbf{i}_{A 1}\right] \times\left[\left(\Sigma_{j} v_{1 j}\right)+\left(\mathbf{u}_{1}-\mathbf{v}_{1}\right) \cdot \mathbf{i}_{E 1}\right]
\end{aligned}
$$

It is symmetric in the interchange of $(A, E)$.

Imagine a change of basis from $\left\{\left(\mathrm{x}_{1}-\mathrm{y}_{1}\right) \cdot \mathbf{i}_{A 1},\left(\mathrm{u}_{1}-\mathrm{v}_{1}\right) \cdot \mathbf{i}_{A 1}\right\}$ to $\left\{\mathbf{q}_{1} \cdot \mathbf{i}_{A 1}, \mathbf{r}_{1} \cdot \mathbf{i}_{A 1}\right\}$. (We do not know the coordinates of this basis change.) Then we have that $\left(S_{A 1} S_{E 1}+T_{A 1} T_{E 1}\right)$ is an inhomogeneous bilinear form in $\left\{\mathbf{q}_{1} \cdot \mathbf{i}_{A 1}, \mathbf{r}_{1} \cdot \mathbf{i}_{A 1}\right\}$ (on one hand) and $\left\{\mathbf{q}_{1} \cdot \mathbf{i}_{E 1}, \mathbf{r}_{1} \cdot \mathbf{i}_{E 1}\right\}$ (on the other hand), again symmetric with respect to $(A, E)$. So we may write it as

$$
\begin{aligned}
\left(S_{A 1} S_{E 1}+T_{A 1} T_{E 1}\right) & =\alpha_{1}+\beta_{1}\left(\mathbf{q}_{1} \cdot \mathbf{i}_{A 1}+\mathbf{q}_{1} \cdot \mathbf{i}_{E 1}\right)+\gamma_{1}\left(\mathbf{r}_{1} \cdot \mathbf{i}_{A 1}+\mathbf{r}_{1} \cdot \mathbf{i}_{E 1}\right)+ \\
& +\delta_{1}\left(\mathbf{q}_{1} \cdot \mathbf{i}_{A 1}\right)\left(\mathbf{q}_{1} \cdot \mathbf{i}_{E 1}\right)+\varepsilon_{1}\left(\mathbf{r}_{1} \cdot \mathbf{i}_{A 1}\right)\left(\mathbf{r}_{1} \cdot \mathbf{i}_{E 1}\right)+ \\
& +\tau_{1}\left[\left(\mathbf{q}_{1} \cdot \mathbf{i}_{A 1}\right)\left(\mathbf{r}_{1} \cdot \mathbf{i}_{E 1}\right)+\left(\mathbf{r}_{1} \cdot \mathbf{i}_{A 1}\right)\left(\mathbf{q}_{1} \cdot \mathbf{i}_{E 1}\right)\right]
\end{aligned}
$$

where we do not know $\alpha_{1}, \beta_{1}, \gamma_{1}, \delta_{1}, \varepsilon_{1}, \tau_{1} \in Z_{p-1}$. (We should not confuse $\alpha_{1}$ with the previously defined $\alpha_{A j}$.)

By repeating with $E_{j}^{\prime}$ instead of $E$, and seeing the dependence of $f_{A E^{\prime}}$ on $\mathbf{q}_{1} \cdot \mathbf{i}_{E_{j}^{\prime}}$ and $\mathrm{r}_{1} \cdot \mathbf{i}_{E_{j}^{\prime}}$, user $A$ can establish the ratios of the coefficients $b \mathrm{f} 1$, $\mathbf{q}_{1} \cdot \mathrm{i}_{E 1}, \mathbf{r}_{1} \cdot \mathbf{i}_{E 1}$, respectively:

$$
\begin{aligned}
& {\left[\alpha_{1}+\beta_{1} \mathbf{q}_{1} \cdot \mathbf{i}_{A 1}+\gamma_{1} \mathbf{r}_{1} \cdot \mathbf{i}_{A 1}\right]:\left[\beta_{1}+\delta_{1} \mathbf{q}_{1} \cdot \mathbf{i}_{A 1}+\tau_{1} \mathbf{r}_{1} \cdot \mathbf{i}_{A 1}\right],} \\
& {\left[\alpha_{1}+\beta_{1} \mathbf{q}_{1} \cdot \mathbf{i}_{A 1}+\gamma_{1} \mathbf{r}_{1} \cdot \mathbf{i}_{A 1}\right]:\left[\gamma_{1}+\tau_{1} \mathbf{q}_{1} \cdot \mathbf{i}_{A 1}+\varepsilon_{1} \mathbf{r}_{1} \cdot \mathbf{i}_{A 1}\right] .}
\end{aligned}
$$

Call this data $\left({ }^{* *}\right)_{A}$. When other collaborators $A^{\prime}, A^{\prime \prime}$ repeat the experiment with their different values of $\mathbf{q}_{1} \bullet \mathbf{i}_{A^{\prime} 1}, \mathbf{r}_{1} \bullet \mathbf{i}_{A^{\prime} 1}, \mathbf{q}_{1} \bullet \mathbf{i}_{A^{\prime \prime} 1}, \mathbf{r}_{1} \cdot \mathbf{i}_{A^{\prime \prime} 1}$, but using the same basis vectors $q_{1}$ and $\mathbf{r}_{1}$, the data $\left({ }^{* *}\right)_{A}$ and $\left({ }^{* *}\right)_{A^{\prime}}$ and $\left({ }^{* *}\right) A^{\prime \prime}$ suffices to calculate the ratios

$$
\alpha_{1}: \beta_{1}: \gamma_{1}: \delta_{1}: \varepsilon_{1}: \tau_{1}
$$

Without loss of generality we can divide through by $\alpha_{1}$ (and change $\hat{\gamma}$ accordingly), and so we calculate

$$
\left(\alpha_{1}=1\right), \beta_{1}, \gamma_{1}, \delta_{1}, \varepsilon_{1}, \tau_{1} .
$$


Repeat with $k=2,3, \ldots, N$ replacing 1 , to calculate

$$
\left(\alpha_{k}=1\right), \quad \beta_{k}, \gamma_{k}, \delta_{k}, \varepsilon_{k}, \tau_{k}, \quad k=1,2, \ldots, N .
$$

Then we have expressed

$$
\begin{aligned}
f_{A E}=\tilde{\gamma} \prod_{j=1}^{N}\left[\alpha_{j}+\beta_{j}\left(\mathbf{q}_{j} \cdot \mathbf{i}_{A j}+\mathbf{q}_{j} \cdot \mathbf{i}_{E j}\right)+\gamma_{j}\left(\mathbf{r}_{j} \cdot \mathbf{i}_{A j}+\mathbf{r}_{j} \cdot \mathbf{i}_{E j}\right)+\delta_{j}\left(\mathbf{q}_{j} \cdot \mathbf{i}_{A j}\right)\left(\mathbf{q}_{j} \cdot \mathbf{i}_{E j}\right)+\right. \\
\left.+\varepsilon_{j}\left(\mathbf{r}_{j} \cdot \mathbf{i}_{A j}\right)\left(\mathbf{r}_{j} \cdot \mathbf{i}_{E j}\right)+\tau_{j}\left\{\left(\mathbf{q}_{j} \cdot \mathbf{i}_{A j}\right)\left(\mathbf{r}_{j} \cdot \mathbf{i}_{E j}\right)+\left(\mathbf{r}_{j} \cdot \mathbf{i}_{A j}\right)\left(\mathbf{q}_{j} \cdot \mathbf{i}_{E j}\right)\right\}\right]
\end{aligned}
$$

with $\tilde{\gamma}$ unknown, and $\alpha_{j}=1$, and the rest of the data $\mathbf{q}_{j}, \beta_{j}$, etc. known. We can compute $\tilde{\gamma}$ from one known key $f_{A_{0} B_{0}}$, so we know the complete expression.

This expression depends only on the public information $\mathbf{i}_{A j}, \mathbf{i}_{E j}$ of $A$ and $E$, so it holds as well for victims $F, E$ :

$$
\begin{aligned}
f_{F E}=\tilde{\gamma} \prod_{j=1}^{N}\left[\alpha_{j}+\beta_{j}\left(\mathbf{q}_{j} \cdot \mathbf{i}_{F j}+\mathbf{q}_{j} \cdot \mathbf{i}_{E j}\right)+\gamma_{j}\left(\mathbf{r}_{j} \cdot \mathbf{i}_{F j}+\mathbf{r}_{j} \cdot \mathbf{i}_{E j}\right)+\delta_{j}\left(\mathbf{q}_{j} \cdot \mathbf{i}_{F j}\right)\left(\mathbf{q}_{j} \cdot \mathbf{i}_{E j}\right)+\right. \\
\left.+\varepsilon_{j}\left(\mathbf{r}_{j} \cdot \mathbf{i}_{F j}\right)\left(\mathbf{r}_{j} \cdot \mathbf{i}_{E j}\right)+\tau_{j}\left\{\left(\mathbf{q}_{j} \cdot \mathbf{i}_{F j}\right)\left(\mathbf{r}_{j} \cdot \mathbf{i}_{E j}\right)+\left(\mathbf{r}_{j} \cdot \mathbf{i}_{F j}\right)\left(\mathbf{q}_{j} \cdot \mathbf{i}_{E j}\right)\right\}\right]
\end{aligned}
$$

and

$$
K_{F E}=\hat{g}^{\left(f_{F E}\right)}
$$

We have used the private information of $2 N+3$ collaborators and the public information $\mathbf{i}_{F j}, \mathbf{i}_{E j}$ of the victims $F, E$, and obtained the key shared by victims $F, E$.

Remark: It is interesting to note that the act of collusion may be even easier than the legitimate use of the system, because the colluders use addition and multiplication, respectively, in $Z_{p-1}$ while the legitimate users are using multiplication and exponentiation, respectively, in $Z_{p}{ }^{*}$.

\section{Example}

Take the simple case $N=1$. Here we can ignore the permutations $\pi_{A}$, which give just minor complications. We have $M=2 N+1=5$ users, $A, B, C, D, E$, colluding to break the scheme. They determine the following $M(M-1) / 2=10$ equations: 


$$
\begin{aligned}
& e_{A B^{c}}^{(1)} c_{A 1}+e_{A B}^{(2)} c_{A 2} \equiv e_{B A}^{(1)} c_{B 1}+e_{B A}^{(2)} c_{B 2} \bmod p-1 \\
& e_{A C^{C} c_{A 1}}^{(1)}+e_{A C^{c}}^{(2)} c_{A 2} \equiv e_{C A}^{(1)} c_{C 1}+e_{C A}^{(2)} c_{C 2} \bmod p-1
\end{aligned}
$$

$$
e_{D E^{c} D 1}^{(1)}+e_{D E}^{(2)} c_{D 2} \equiv e_{E D^{c}}^{(1)} c_{E 1}+e_{E D}^{(2)} c_{E 2} \bmod p-1
$$

Notice that $e_{A B}^{(1)}=Z_{A, 1,1}^{(B)}$.

Starting with the $[M(M-1) / 2] \times[M(N+1)]=10 \times 10$ matrix of coefficients $\pm e_{A B}^{(1)}$, delete one column to make it an inhomogeneous system, and delete one row to make it square. We obtain the $9 \times 9$ matrix $F$ :

$\left[\begin{array}{ccccccccc}e_{A B}^{(1)} & e_{A B}^{(2)} & -e_{B A}^{(1)} & -e_{B A}^{(2)} & 0 & 0 & 0 & 0 & 0 \\ e_{A C}^{(1)} & e_{A C}^{(2)} & 0 & 0 & -e_{C A}^{(1)} & -e_{C A}^{(2)} & 0 & 0 & 0 \\ 0 & 0 & e_{B C}^{(1)} & e_{B C}^{(2)} & -e_{C B}^{(1)} & -e_{C B}^{(2)} & 0 & 0 & 0 \\ 0 & 0 & e_{B D}^{(1)} & e_{B D}^{(2)} & 0 & 0 & -e_{D B}^{(1)} & -e_{D B}^{(2)} & 0 \\ 0 & 0 & 0 & 0 & e_{C D}^{(1)} & e_{C D}^{(2)} & -e_{D C}^{(1)} & -e_{D C}^{(2)} & 0 \\ 0 & 0 & 0 & 0 & e_{C E}^{(1)} & e_{C E}^{(2)} & 0 & 0 & -e_{E C}^{(1)} \\ 0 & 0 & 0 & 0 & 0 & 0 & e_{D E}^{(1)} & e_{D E}^{(2)} & -e_{E D}^{(1)} \\ -e_{A D}^{(1)} & -e_{A D}^{(2)} & 0 & 0 & 0 & 0 & e_{D A}^{(1)} & e_{D A}^{(2)} & 0 \\ -e_{A E}^{(1)} & -e_{A E}^{(2)} & 0 & 0 & 0 & 0 & 0 & 0 & e_{E A}^{(1)} \\ & & & & & & & & \end{array}\right]$

Define vectors $\mathbf{C}, \mathbf{e}$ as

$$
\mathrm{C}=\left(c_{A 1}, c_{A 2}, c_{B 1}, c_{B 2}, c_{C 1}, c_{C 2}, c_{D 1}, c_{D 2}, c_{E 1}\right)^{\mathrm{T}}
$$




$$
\mathbf{e}=\left(0,0,0,0,0, e_{E C}^{(2)}, e_{E D}^{(2)}, 0,-e_{E A}^{(2)}\right)^{\mathrm{T}} .
$$

We are then faced with solving the $9 \times 9$ system of inhomogeneous linear equations $F \mathrm{C}=\mathrm{ec}_{E 2}$. To facilitate the solution of this system, we depend on $F$ having full rank, that is, $\operatorname{det}(F)$ is a unit in $Z_{p-1}$. To verify that $F$ generally has full rank, we perform some elementary column operations. Letting $F_{j}$ denote the $j$ th column, we set $F_{2} \leftarrow\left(c_{A 1} F_{1}+c_{A 2} F_{2}\right) \hat{y} / \gamma$, $F_{4} \leftarrow\left(c_{B 1} F_{3}+c_{B 2} F_{4}\right) \hat{\gamma} / \gamma, \quad F_{6} \leftarrow\left(c_{C 1} F_{5}+c_{C 2} F_{6}\right) \hat{\gamma} / \gamma, \quad$ and $F_{8} \leftarrow\left(c_{D 1} F_{7}+c_{D 2} F_{8}\right) \hat{y} / \gamma$. The new entry in the first row, second column, is now

$$
\begin{aligned}
& \left(c_{A 1} e_{A B}^{(1)}+c_{A 2} e_{A B}^{(2)}\right) \hat{y} / \gamma=\left(c_{A 1} Z_{A 11}^{(B)}+c_{A 2} Z_{A 12}^{(B)}\right) \hat{\gamma} / \gamma \\
& =\gamma\left(S_{A 1} S_{B 1}+T_{A 1} T_{B 1}\right) \hat{\gamma} / \gamma=\hat{\gamma}\left(S_{A 1} S_{B 1}+T_{A 1} T_{B 1}\right)=f_{A B},
\end{aligned}
$$

recalling $f_{A B}=\hat{\gamma}\left(S_{A 1} S_{B 1}+T_{A 1} T_{B 1}\right)=f_{B A}$. The altered matrix is then seen to be

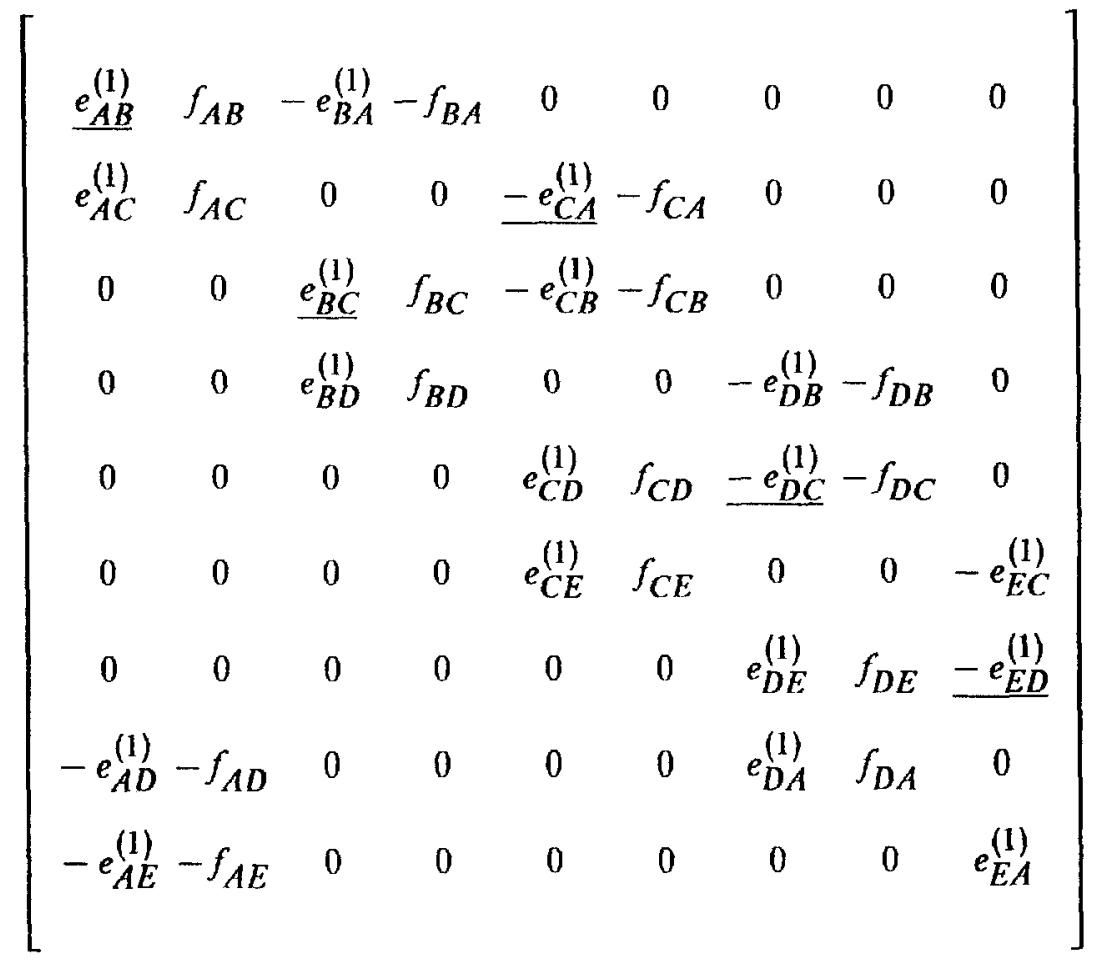

Consider the five underlined entries. A typical one is 


$$
e_{B C}^{(1)}=r_{B 11}\left(S_{B 1} S_{C 1}+T_{B 1} T_{C 1}\right)+\alpha_{B 11} \cdot \mathrm{i}_{C 1}+\beta_{B 11} \cdot \mathrm{i}_{C 1}^{c}
$$

It depends on the random vector $\alpha_{B 11}$, as do the other entries $e_{B X}^{(1)}$ in that column. But the dot product $\alpha_{B 11}{ }^{\circ} i_{C 1}$ is linearly independent of the other such products $\alpha_{B 11} \bullet \mathbf{i}_{X 1}$. So, by varying $\alpha_{B 11}$, the entry $e_{B C}^{(1)}$ can vary without changing any other matrix entries. (Note: $e_{B C}^{(2)}$ also depended on $\alpha_{B 11} \cdot i_{C 1}$, through the dependence between $\alpha_{B 11}$ and $\alpha_{B 12}$. But $k_{B C}$ is independent of the choice of $\alpha_{B 11}$. This is why we did the elementary column operations, to remove this interdependence between entries.)

The cofactor of the five underlined entries is the $4 \times 4$ matrix

$$
\left[\begin{array}{cccc}
0 & f_{B D} & 0 & -f_{D B} \\
0 & 0 & f_{C E} & 0 \\
-f_{A D} & 0 & 0 & f_{D A} \\
-f_{A E} & 0 & 0 & 0
\end{array}\right]
$$

whose determinant, $f_{B D} f_{C E} f_{A D} f_{A E}$ is in general nonzero. Thus $\operatorname{det}(F)$ is not identically 0 . This gives evidence that the linear equations will usually be solvable.

It may become necessary to solve the equations $\bmod q$ for each prime $q$ dividing $p-1$, and piece together with the Chinese remainder theorem. In particular, for small primes $q$ the determinant might vanish accidentally and we might have to compute logarithms $\bmod q$ by brute force.

Consider now the general case $N \geq 1$. Now the general matrix entry $e_{A B}^{(k)}$ is not just $Z_{A, k, j}^{(B)}$ but rather a sum of products of such $Z$ values. Nonetheless there are enough randomly chosen values $\alpha_{A j \ell}$ that an independence argument can be made similar to the case $N=1$.

Select $M \geq 2 N+1$ conspirators randomly, and build an $[M(M-1) / 2] \times[M(N+1)]$ matrix $F$ corresponding to the example $F$; we have again omitted one column to make an inhomogeneous system, but this time we do not drop any rows. Also $M$ may be somewhat larger than $2 N+3$.

If the rank of $F$ is fully $M(N+1)-1$, we solve as before. 
Suppose that, as $M$ grows, the rank of $F$ is always at least $M(N+1)-I$, where $I$ is independent of $M$. Then, instead of representing all $c_{A i}$ as multiples of a single $c_{\underline{B} 1}$ and using the corresponding group element $\hat{g}$, we select $I$ different reference points $c_{\underline{B} i}$ and use corresponding group elements $\hat{g}_{i}$. This is more tedious but still possible. One or two more conspirators might be necessary in this case.

Suppose that the rank of $F$ is always bounded by $M(N+1-J), J \geq 1$. This suggests that $J$ linear relations exist among the $N+1$ columns of $e_{A X}^{(i)}$ corresponding to each user $A$, and that the quantities $c_{A i}$ could vary with $J$ degrees of freedom without affecting the keys. (This would mean that the quantities described as independent variables were actually being chosen in a dependent fashion.) Detect these linear combinations and describe the system in terms of a reduced set of $N+1-J$ variables $c_{A i}$. Then continue as before.

Finally, any systematically low rank of $F \bmod q$ would indicate systematic relations among the keys $K_{A B}$, which we should be able to exploit directly.

\section{References}

1. W. Diffie and M. E. Hellman, "New directions in cryptography," IEEE Trans. Informat. Theory, vol. IT-22, pp. 644-654, Nov. 1976.

2. S. Tsujii, K. Araki, and T. Sekine, "A new scheme of non interactive ID-based key sharing with explosively high degree of separability (second version)," Technical report of Department of Computer Science, Tokyo Institute of Technology, 93TR-0020, July 1993.

Note: the NIKS-TAS scheme has since appeared in the 1993 Korea-Japan Joint Workshop on Information Security and Cryptology. A partial attack, in the same workshop, was "An Attack on an ID-Based Key Sharing System," by V. Luchangco and K. Koyama. 